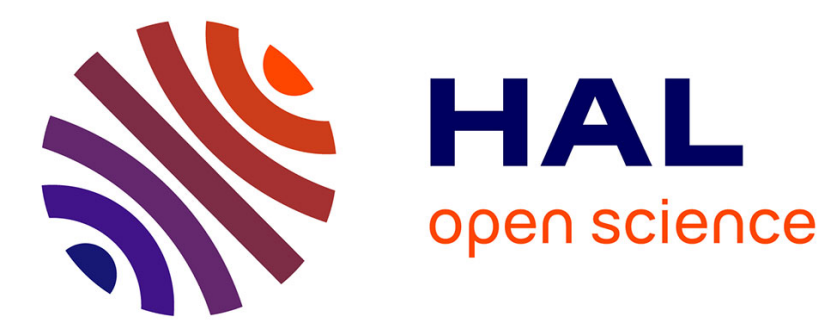

\title{
L'encadrement de l'abattage rituel industriel dans l'union européenne: limites et perspectives
}

\author{
Florence Bergeaud-Blackler
}

\section{To cite this version:}

Florence Bergeaud-Blackler. L'encadrement de l'abattage rituel industriel dans l'union européenne: limites et perspectives. Politique européenne, 2008, 24, pp.103-122. halshs-00178667

\section{HAL Id: halshs-00178667 https://shs.hal.science/halshs-00178667}

Submitted on 3 Jul 2008

HAL is a multi-disciplinary open access archive for the deposit and dissemination of scientific research documents, whether they are published or not. The documents may come from teaching and research institutions in France or abroad, or from public or private research centers.
L'archive ouverte pluridisciplinaire HAL, est destinée au dépôt et à la diffusion de documents scientifiques de niveau recherche, publiés ou non, émanant des établissements d'enseignement et de recherche français ou étrangers, des laboratoires publics ou privés. 


\section{Florence BERGEAUD-BLACKLER}

\section{L'ENCADREMENT DE L'ABATTAGE RITUEL INDUSTRIEL DANS L'UNION EUROPÉENNE : LIMITES ET PERSPECTIVES}

Dans nos sociétés sécularisées, il existe quelques domaines où la tentation du politique de réguler le champ religieux n'a pas totalement disparue. C'est le cas de l'industrie d'abattage en Europe. Les aliments religieux sont de plus en plus nombreux à circuler dans la chaîne alimentaire conventionnelle posant la question de leur double statut d'aliment et de produit religieux. Alors qu'elle s'apprête à réviser la directive sur l'abattage, l'Union Européenne pourra-t-elle faire l'économie d'une réflexion sur la régulation des aliments religieux? L'occasion pour nous d'en saisir les principaux enjeux.

Pour citer cet article veuillez-vous référer à la version définitive parue dans la revue Politique Européenne, №24, hiver 2008 Dieu loin de Bruxelles. L'européanisation informelle du religieux - sous la direction de François Foret et Xabier Itçaina.

Dans nos sociétés sécularisées, il existe quelques domaines où la tentation du politique de réguler le champ religieux n'a pas totalement disparue. C'est le cas de l'industrie d'abattage. La plupart des législations européennes encadrent l'abattage " selon un rite religieux " et le dispense ainsi de l'obligation faites aux autres modes d'abattage d'étourdir les animaux avant leur mise à mort. Or, au sein des instances religieuses, la question de l'acceptabilité des méthodes d'insensibilisation n'est pas définitivement tranchée. Prévoir une dérogation publique à l'obligation d'insensibiliser les animaux d'abattage revient donc à reconnaitre une particularité rituelle et donc en quelque sorte à légitimer une option religieuse, altérant du même coup la dynamique interne et la portée des débats dans la sphère religieuse. Plus encore et c'est ce que nous voulons montrer ici, en légiférant partiellement et en ne s'assurant pas de la réelle destination des carcasses issues de l'abattage rituel, les États européens ayant adopté cette dérogation consolidée par une directive européenne, ont laissé les acteurs économiques empiéter le champ normatif religieux, brouillant les frontières du religieux, du politique et de l'économique. Destiné à produire une viande pour des minorités religieuses, l'abattage religieux est devenue pratique courante dans les abattoirs de nombreux pays d'Europe sans considération de la destination finale des carcasses. La surproduction de carcasses rituellement abattues permet de satisfaire à la fois les marchés rituels et les marchés conventionnels, la loi ne faisant pas obligation de les réserver à la seule consommation religieuse. Reste une question que personne ne souhaite vraiment soulever alors même que la traçabilité est au cœur des préoccupations de la gouvernance alimentaire: "qu'en penseraient les consommateurs s'ils savaient »? 
Un rapport non publié de l'administration publique française estimait qu'en France métropolitaine les quatre cinquièmes des ovins abattus transitaient par un circuit d'abattage rituel, une part importante des viandes ainsi produites débouchant vers des circuits de distribution conventionnels sans étiquetage spécifique ${ }^{1}$. Des situations identiques existeraient dans d'autres pays européens mais la "sensibilité du sujet " expliquerait qu'on ne communique pas les véritables dimensions du problème ${ }^{2}$.

Aujourd'hui, la révision par les services de la Commission de la directive européenne de $1993^{3}$ est l'occasion de relancer la bataille sur l'harmonisation des méthodes et la traçabilité des viandes rituelles, bataille dont un premier épisode s'est déroulé de 1991 à 1993 au sein des institutions communautaires. D'une part parce que l'approche de la directive de 1993 à ce sujet est devenue, sur ce point particulier, incohérente avec la nouvelle approche « de la ferme à la table » prônée par les institutions communautaires. D'autre part, parce que la situation économique et sociale qui expliquait les orientations prises dans la directive 1993 a changé sur de nombreux points.

Après une description et une mise en perspective de son encadrement dans l'Union européenne, nous revenons sur les débats très largement méconnus qui ont eu lieu lors de la fabrication de la directive de 1993, puis nous essayons de comprendre ce qui a changé et comment une autre approche parait s'imposer aujourd'hui plus en cohérence avec le projet politique de construction européenne. La politique de transparence de l'Union Européenne conduira-t-elle aujourd'hui à séculariser les abattoirs, c'est-à-dire finalement à aboutir à une division claire des tâches et des responsabilités religieuses, économiques et politiques?

\section{L'encadrement de l'abattage rituel ${ }^{4}$ industriel et de ses produits}

Pratique réservée à une partie des minorités juives d'Europe jusqu'au milieu du $\mathrm{xx}^{\mathrm{e}}$ siècle, concerne désormais un nombre important de citoyens européens. Les deux

\footnotetext{
* Je remercie les services des archives de la Commission européenne, et du Conseil de l'Union européenne, ainsi que l'Eurogroup for animal pour leur efficacité dans la mise à disposition des documents sollicités. Mes remerciements vont également à $\mathrm{M}$. Denis Simonin pour ses commentaires ainsi qu'à M. Guigui, Grand Rabbin de Bruxelles pour l'entretien qu'il nous a accordé.

${ }^{1}$ Babre Simon, Berloz Jean-Marc, Riera Ramiro et al. (2005), " Le champ du halal ", Rapport d'activité 2005, mission du COPERCI, ministère de l'Intérieur et de l'Aménagement du territoire, ministère de l'Agriculture et de la Pêche, Inspection générale de l'Agriculture, p. 65-67, septembre. Le rapport n'a pas été rendu public mais ses conclusions sont accessibles sur le site du Ministère de l'Agriculture :

[http://agriculture.gouv.fr/sections/publications/rapports/rapport-d-activite-2005-de-l-inspection-generale-de-lagriculture/downloadFile/FichierAttache_1_f0/rapport_activte_iga1.pdf?nocache=1134040585.85].

${ }^{2}$ II est rare que les industriels de la viande reconnaissent qu'ils abattent en mode rituel des viandes destinées à des marchés non musulmans. C'est le cas toutefois de la Danish Meat Association: "Danish poultry is halal slaughtered because large quantities are exported to Muslim countries. It is not known at the time of slaughter where the poultry is to be sold. [...] It is therefore most practical to halal slaughter the complete production". [http://www.danishmeat.dk/smcms/13068/13212/13226/13227/Index.htm?ID=13227], "Halal Slaughter", consulté le 26 septembre 07.

${ }^{3}$ Directive 93/119/CE du Conseil du 22 décembre 1993 sur la protection des animaux au moment de leur abattage ou de leur mise à mort. (JO L 340 du 31.12.1993, p. 21-34).

${ }^{4}$ Dans cet article, les expressions « abattage rituel ", " abattage selon un rite religieux ", " abattage religieux » sont interchangeables. Pour plus de commodité nous les remplaçons par les initiales AR.
} 
principales opérations de mise à mort selon un rite religieux ${ }^{5}$ effectuées dans un cadre industriel sont la shekhita et le dhakât (cf. encadré) donnant lieu à des viandes respectivement casher ${ }^{6}$ et halal ${ }^{7}$. Ils intéressent en premier lieu, mais pas nécessairement exclusivement, les minorités musulmanes et juives d'Europe estimées à plus de 21 millions (plus de 90 millions avec la Turquie) pour les premières, et à près de 1,5 million pour les secondes, pour une population européenne totale d'environ 525 millions d'habitants hors-Turquie et 595 millions avec la Turquie ${ }^{8}$.

\section{La shekhita}

La shekhita, c'est-à-dire l'AR des animaux selon la loi juive destiné à produire une viande casher, peut être décrite à travers les 4 étapes qui la composent : l'examen, la prise de l'animal, l'incision, et enfin l'inspection. L'examen visuel de l'animal permet au shohet d'écarter les animaux qui échoueront probablement au contrôle postmortem de la carcasse. La seconde étape consiste à préparer l'acte d'incision en contenant l'animal - ce qui en abattoir se fait grâce à un box de contention ou piège spécialement adapté à l'AR - et à affûter la lame selon un geste technique précis. L'incision constitue la troisième étape, son objectif est en théorie de réduire la souffrance animale par l'emploi d'une technique précise et rapide. Enfin, la quatrième et dernière étape consiste en une bedika, c'est-à-dire un contrôle de certaines parties de la carcasse et des principaux viscères et à l'éventuel retrait des carcasses non conformes. Les professions d'abatteur (shohet) et de contrôleurs (bodek) sont instituées et contrôlées par un tribunal rabbinique. Le titulaire n'est pas propriétaire de son habilitation comme shohet. Une faute professionnelle peut en principe aboutir à un retrait temporaire ou définitif de son habilitation.

\footnotetext{
${ }^{5}$ Un troisième type d'abattage rituel est pratiqué en Europe (Royaume-Uni en particulier) : le Jhakta, méthode essentiellement réalisée dans le cadre du Sikhisme consistant à égorger l'animal par décapitation (ovins et caprins).

${ }^{6}$ Casher : terme hébraïque désignant les aliments jugés « aptes » ou propre à la consommation, conformément aux lois de l'alimentation (cacherout) bibliques et rabbiniques (Wigoder, Goldberg et al., 1996). S'écrit indifféremment cacher ou casher dans sa version française, sans italique.

${ }^{7} \mathrm{Halal}$ : terme arabe traduit par licite. S'écrit indifféremment hallal ou halal en français, sans italique.

${ }^{8}$ Calcul réalisé d'après les données du 2006 Report on International Religious Freedom (2006).

${ }^{9}$ Prononcer « chrita».
} 


\section{Le dhakât}

Par comparaison, le dhakât musulman effectué dans un contexte industriel pour produire une viande halal, est plus simple que la shekhita puisqu'il ne comporte aucun contrôle pré- et postmortem de l'animal et de la carcasse, le dhakât peut être effectué soit par dhabiha, c'est-à-dire par égorgement soit par nahr $r^{11}$. Rien n'est formellement spécifié concernant l'instrument utilisé, la technique d'affûtage de la lame, les moyens de réduire la souffrance animale étant laissés à l'appréciation des abatteurs musulmans. La fonction d'abatteur musulman n'est pas religieusement institutionnalisée. En principe, n'importe quel homme ou femme, peut la pratiquer à condition de respecter des critères de foi, de conscience, et de moralité. Enfin, autre différence d'importance, la chaîne d'abattage industrielle en mode musulman ne rejette rien, c'est uniquement l'égorgement rituel qui rend la carcasse licite.

En ce qui concerne l'encadrement réglementaire du rite d'abattage, son périmètre diffère selon que l'AR est considéré en tant que mode de production à visée commerciale à l'exemple des États-Unis, ou bien une pratique religieuse comme c'est plutôt le cas en Europe aujourd'hui.

\section{L'approche consumériste américaine}

Pour la Food and Drug Administration (FDA) « le terme casher ne devrait être utilisé que pour les produits alimentaires conformes à certaines prescriptions religieuses " (Gutman, 1999; CSPI, 1998). La formulation très vague de l'agence fédérale s'accommode toutefois de définitions beaucoup plus précises dans certains États américains. Dans vingt-six d'entre eux les viandes rituelles ne peuvent porter cette appellation que sous des conditions strictes validées par les pouvoirs publics. Ainsi, pour prévenir les abus, les Jewish dietary laws ${ }^{12}$ prohibent toute publicité ou vente de produits présentés comme casher qui ne seraient pas conformes aux procédés de fabrication et manipulation que ces laws décrivent. L'État de New York, le premier à se doter d'une loi sur l'alimentation casher (1915) a inspiré au fil du temps vingt-cinq autres États qui l'ont adaptée individuellement (Masoudi, 1993, 671). On distingue aujourd'hui deux grands groupes de Kosher food laws. Dans vingt-deux États, le terme kosher est défini selon les préceptes stricts des juifs orthodoxes, dans quatre autres les kosher fraud laws font référence de façon plus générale à la tradition juive sans allusion spécifique à un courant religieux (Masoudi, 1993, 672). Certains d'entre eux mettent en place des conseils rabbiniques afin de les conseiller, d'effectuer la surveillance ou encore le contrôle (Marei, 2001, 23). Au nom de la transparence, de la défense du consommateur et de la concurrence, les pouvoirs publics interviennent assez

\footnotetext{
${ }^{10}$ Prononcer « zakate ». Nous utilisons le terme dhakât qui nous semble plus précis que l'expression abattage halal habituellement utilisée. Le dhakât signifie « immolation rituelle », cf. Benkheira Hocine, « Le rite à la lettre. Régime carné et normes religieuses ", Pierre Bonte, Anne Marie Brisebarre et Altan Gökalp (dir.), Sacrifices en Islam: espaces et temps d'un rituel, Paris, CNRS Éditions, Paris, CNRS Éditions, 1999, coll. "CNRS Anthropologie ", p. 70.

${ }^{11}$ Le nahr est une technique plutôt réservée aux grands mammifères, la saignée est obtenue en enfonçant verticalement une lame au niveau de la base du coup.

${ }^{12}$ Egalement appelées Kosher food laws ou Kosher fraud laws, ou encore kosher dietary laws.
} 
directement dans le champ religieux. Ceci a toutefois suscité de nombreuses critiques (Gutman, 1999, 2369), mais jusqu'à aujourd'hui les procédures demandant l'abolition de ces lois ont été majoritairement déboutées sur la base de l'argument selon lequel ces lois ne seraient pas hostiles à la liberté religieuse, mais au contraire en garantiraient la possibilité (Masoudi, 1993 ; Marei, 2001).

La première halal food law a été établie dans le New Jersey $(2000)^{13}$ selon le modèle d'une kosher food law, distinct de celui de l'État de New York, et destiné à échapper à l'argument d'anticonstitutionnalité. Le modèle du New Jersey s'en distingue en ce qu'aucune définition n'est donnée des termes kosher et halal, a priori l'Etat s'engage à contrôler la conformité des produits sur la base des standards élaborés par les détaillants eux-mêmes. "Retailers are permitted to set their own standard of what is identified as kosher. They are required to post a sign, provided by the Division, which details their understanding of the term "kosher". The sign must be visible to all consumers. Additionally, if the retailer has rabbinical certification, a second sign must be posted which identifies the supervising rabbi and the number of visits that have been made. The Bureau investigates and enforces only the claims made by the retailer" ${ }^{\prime 4}$. Avec le modèle New Jersey, les dietary laws mettent en avant la responsabilité des commerçants devant l'État qui se contente de contrôler les acteurs économiques, lesquels définissent leurs standards de production en suivant éventuellement un modèle religieux. L'objectif de protection des consommateurs est atteint mais ce modèle a un inconvénient majeur, celui de réduire considérablement la portée du travail " légitime » des autorités religieuses sur la définition du halal.

\section{L'encadrement par l'UE : objectif économique, respect des droits fondamentaux, prise en compte du bien-être animal}

À l'exception de l'Espagne qui a mis en place un partenariat privilégié avec l'Instituto Halal, aucun État européen n'a établi de standard casher ou halal. Sur le plan de l'UE, il n'existe pas de food laws pour les aliments rituels, ceux-ci étant soumis aux mêmes conditions de production et d'identification que les autres aliments. Toutefois les institutions communautaires prennent en compte le caractère particulier de l'abattage religieux et lui reconnaissent un droit à se soustraire à certaines obligations, et notamment à celle d'étourdir l'animal avant sa mise à mort. Mais elles ne prévoient pas que les viandes ainsi produites soient étiquetées comme telles. La raison en serait que leur caractéristique "religieuse » les positionnerait en dehors du champ d'intervention communautaire. Selon les fonctionnaires européens interrogés, il serait en quelque sorte naturel que l'Europe, continent sécularisé, ne s'occupe pas de réguler des produits religieux. Pourtant, même si peu de publicité a été faite autour de cela, l'étiquetage des aliments rituels et une harmonisation minimale des pratiques

\footnotetext{
${ }^{13}$ Buying halal Food, brochure publiée par le New Jersey Office of the Attorney General, Division of Consumer Affairs, Office of Consumer Protection, Kosher Food Enforcement Unit, [http:/www.state.nj.us/ps/ca/brief/halal.pdf].

${ }^{14}$ Buying Kosher Food, "Regulations Governing the Sale of Food Represented as Kosher", brochure publiée par le New Jersey Division of Consumer Affair, [http://www.njconsumeraffairs.gov/brief/kosher.pdf].
} 
religieuses d'abattage ont bien été envisagés lors de la rédaction de la directive de 1993 à ce sujet encore en vigueur aujourd'hui ${ }^{15}$.

Avant de rapporter plus en détail les discussions qui ont eu lieu autour de ce que nous appellerons par commodité «la directive de 1993 », précisons d'abord en quoi les institutions communautaires sont concernées par la régulation de l'AR.

La libre circulation des marchandises dans I'UE est un des principaux objectifs du traité de l'UE et son organisation une de ses premières priorités. Dans les années quatre-vingt, l'harmonisation des standards de production et des étiquetages des denrées alimentaires ont constitué des mesures phares de l'achèvement du marché unique (1992). Comme son titre ne l'indique pas, le premier objectif de la directive 1993 " sur la protection des animaux au moment de leur abattage ou de leur mise à mort " est avant tout de parvenir à une libre concurrence non faussée sur le marché unique par l'établissement de normes communes " afin d'assurer un développement rationnel de la production et de faciliter l'achèvement du marché intérieur des animaux et des produits ». II s'agit d'harmoniser autant que possible les pratiques d'abattage afin que les mesures prises pour la santé et la protection des animaux n'agissent pas comme des barrières techniques aux échanges. L'objectif est donc économique avant tout, le bien-être animal n'étant pas un objectif du traité ${ }^{16}$.

La priorité accordée à la libre circulation des produits est toutefois limitée par un droit, celui « d'autoriser des expériences techniques et scientifiques et de prendre en compte les exigences particulières de certains rites religieux » comme le rappelle un considérant de la directive 1993. Cette limitation a été demandée par les États membres au nom des droits fondamentaux par la suite énoncés dans la Charte des Droits fondamentaux, et notamment de l'article 21 qui enjoint les États membres d'interdire toutes discriminations fondées sur la religion ou les convictions, les opinions politiques ou toute autre opinion.

Dans ce contexte la justification de l'encadrement de l'AR par l'UE est triple : d'une part assurer que les pratiques d'abattage, y compris rituelles, n'agissent pas comme barrières techniques aux échanges, qu'elles ne constituent pas des mesures protectionnistes déguisées, d'autre part assurer la liberté religieuse par la prise en compte des rites religieux et lutter par là-même contre les discriminations, et enfin prendre en compte le bien-être animal.

\section{Le dynamisme du marché révèle les limites de la réglementation en la matière}

\section{Recherche du profit, défiance des consommateurs et surenchère du halal}

Les groupes religieux peuvent sur une base volontaire organiser une traçabilité des produits issus de l'AR. C'est le cas le plus fréquent dans la communauté juive où les groupes religieux organisent eux-mêmes un contrôle de la cacheroute, de l'abattoir à

\footnotetext{
${ }^{15}$ Directive 93/119/CE du Conseil, du 22 décembre 1993 (op. cit.). En cours de révision par les services de la Commission.

${ }^{16}$ Un arrêt de la Cour du 12 juillet 2001 (H. Jippes e.a. Affaire C-189/01 Appréciation de la Cour) rappelle en effet que " assurer le bien-être des animaux ne fait pas partie des objectifs du traité (de l'UE), tels qu'ils sont définis à l'article 2 CE [...] une telle exigence n'est (d'ailleurs) pas mentionnée à l'article 33 CE, qui décrit les objectifs de la politique agricole commune " La décision 78/923/CEE du Conseil concernant la conclusion de la Communauté européenne sur la protection des animaux dans les élevages précise également que «la protection des animaux ne constitue pas en soi l'un des objets de la Communauté ».
} 
l'assiette du consommateur (Bauer, 1996 ; Nizar, 1997), une responsabilité qui ne leur a jamais été contesté ${ }^{17}$. II en est autrement pour la production halal. Les acteurs économiques sont les principaux organisateurs et bénéficiaires de ces circuits de production et leur stratégie consiste plutôt à essayer de minimiser la contrainte rituelle. L'absence de consensus religieux sur une définition du halal, un marché fortement compétitif, et la facilité d'obtention d'un permis d'abattage favorisent les stratégies marketing au dépend du travail religieux. Desserrement de la contrainte rituelle ou au contraire surenchère sont les deux conséquences de l'emprise et de la domination du secteur économique sur cette norme.

Dans le premier cas, les abatteurs sont tentés de réduire les opérations spécifiques requises en cas d'AR afin de diminuer le ralentissement des cadences d'abattage (comme réduire le temps d'attente requis entre l'immobilisation de l'animal et la saignée rituelle, en n'utilisant pas le matériel de contention requis). Ils peuvent également desserrer la contrainte rituelle en faisant travailler des abatteurs peu scrupuleux au poste de sacrificateur. Ces pratiques ne sont pas illégales puisque la procédure d'abattage n'est pas définie et protégée. Mais elles ne correspondent pas à l'idée que se font du rituel d'abattage les consommateurs de ces produits, ce qui crée un climat de méfiance et porte ces derniers à demander un resserrement maximum de la contrainte rituelle nonobstant leurs convictions religieuses. Ce qui conduit, en l'absence d'encadrement religieux, vers une " surenchère » de la norme halal.

Pour attirer ce nombre croissant de consommateurs méfiants, certains abatteurs sont tentés de privilégier une conception plus stricte du halal et, pour certains, d'offrir des garanties maximum en abattant la totalité des bêtes en mode rituel quelle que soit la destination de la viande. Les acteurs sont certes en conformité avec la réglementation puisque rien ne les oblige à signaler que ce qu'ils vendent en circuit conventionnel provient d'un AR, mais ils ne respectent pas l'esprit de la loi puisque, comme le leur rappellent les organisations de protection animale, les abattages rituels font l'objet de dérogations spécifiques pour répondre à un besoin religieux.

\section{Figure 1 : Dilemme de la production rituelle dans le modèle européen}

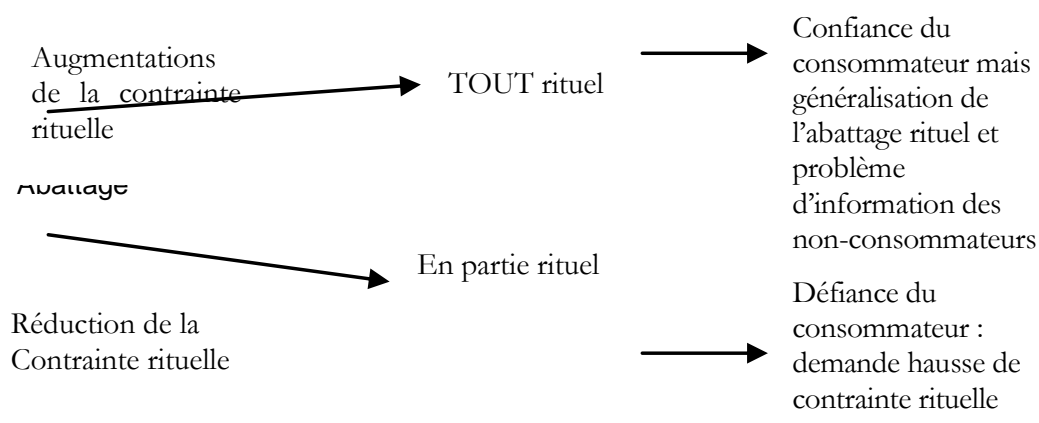

\footnotetext{
${ }^{17}$ Que les religieux soient compétents et responsables dans la gestion de la cacheroute et de ses revenus n'a jamais été contesté, mais que certains groupes s'en attribuent le monopole, voilà qui provoque de nombreuses réactions (Bauer, 1996).
} 
Des qualités sanitaires garanties en principe, mais un problème de traçabilité et d'information

En accordant une dérogation à l'abattage religieux, les pays européens concernés entendent bien garantir à tous les consommateurs, y compris ceux qui mangent halal et casher, les mêmes mesures d'hygiène, de sécurité sanitaire, les mêmes garanties morales relatives au traitement des animaux. Mais l'information sur la qualité religieuse est jugée peu fiable par les acheteurs de produits halal puisque les acteurs économiques la produisent eux-mêmes sans contrôle par un organisme réellement indépendant. Quant aux autres consommateurs, ils ne bénéficient plus de la garantie apportée par la réglementation d'épargner à l'animal " toute douleur ou souffrance évitable » (justification publique de l'étourdissement ${ }^{18}$ ) puisque certaines des viandes qu'ils mangent ne proviennent pas d'animaux étourdis avant leur saignée. Enfin, il y a un problème de cohérence réglementaire car si la viande issue d'AR est orientée vers un circuit de distribution conventionnel, la pratique d'abattage à son origine n'a plus d'objectif religieux, et il en ressort que la dérogation à l'étourdissement ne se justifie plus.

\section{Subsidiarité ou harmonisation : histoire d'un compromis fragile}

L'expression "abattage religieux» est introduite dans le corpus réglementaire communautaire pour la première fois en 1974 par une directive qui rend obligatoire l'étourdissement des animaux sauf dans le cas des abattages religieux (article 4) ${ }^{19}$. Elle est abrogée et remplacée par la directive de 1993, élargie chargée de reprendre les principes établis dans la Convention européenne sur la protection des animaux d'abattage du 10 mai 1979 (que je nommerai ici convention) promulguée par le Conseil de l'Europe à Strasbourg et signée par, une majorité de ses membres ${ }^{20}$. En matière d'AR, cette convention prévoit que les États peuvent " autoriser des dérogations aux dispositions relatives à l'étourdissement préalable dans certains cas dont les abattages selon les rites religieux " (article 17), elle recommande également aux États les autorisant de s'assurer de l'habilitation des sacrificateurs par des organismes religieux dans la mesure où ils ne délivrent pas eux-mêmes les autorisations nécessaires (art. 19).

Initialement, le règlement de 1991 qui donna lieu à la directive de 1993 avait pour but de réunir en un seul texte les principales lignes de la convention déjà approuvée par les pays membres afin que la Communauté européenne puisse la signer. Mais les discussions ont été longues et difficiles, notamment lorsqu'il a été question de l'AR. La reconstitution de ces débats à partir d'archives de la Commission, du Conseil, et de l'Eurogroup for animals ${ }^{21}$, va nous permettre de saisir ici les enjeux de l'encadrement

\footnotetext{
${ }^{18}$ Cette justification réglementaire a également une assise sociale, représentée par les ONG de protection animale et vétérinaire européennes.

${ }^{19}$ Directive 74/577/CEE du Conseil, du 18 novembre 1974, relative à l'étourdissement des animaux avant leur abattage, Journal officiel, $n^{\circ} \mathrm{L} 316$ du 26/11/1974, p. 0010-0011.

20 Certains pays comme la France, l'Espagne et le Royaume-Uni ne l'ont toutefois pas ratifié. [http://conventions.coe.int/Treaty/Commun/ChercheSig.asp?NT=102\&CM=1\&DF=\&CL=FRE].

${ }^{21}$ Autrefois Intergroup on the Welfare and Conservation of Animals créé en 1983 au Parlement européen.
} 
réglementaire communautaire. Le temps ayant été un paramètre important de la négociation, nous les résumons dans l'ordre chronologique de la procédure de « consultation $»^{22}$.

\section{Texte initial de la Commission}

Pour rédiger la première version de règlement les rédacteurs de la Commission ne s'en sont pas tenus au texte de la convention mais ont été bien au-delà en proposant l'harmonisation de certaines opérations spécifiques à l'AR, et l'identification comme telle des viandes qui en sont issues. Le projet de règlement daté du 17 Avril 1991, signé par I'Irlandais Ray Mac Sharry (Fianna Fail) alors commissaire en charge de l'Agriculture ${ }^{23}$, rappelle les objectifs de fluidité des échanges sur le marché unique : "Considérant que tous les États membres autorisent l'abattage rituel, qu'il est nécessaire d'établir des normes minimales pour assurer la libre circulation des produits obtenus par ce type d'abattage " ( $5^{\mathrm{e}}$ considérant). Le point 4 de l'annexe $\mathrm{E}$ de la directive prévoit également que les États membres en assurent la traçabilité : "L'autorité compétente doit veiller à ce que toute la viande produite dans le cadre de l'abattage rituel et destinée à être vendue comme telle soit marquée de manière à pouvoir être identifiée par le consommateur final ${ }^{24}$. Il prévoit en outre des précautions spécifiques avant, pendant et après l'abattage religieux. L'annexe $\mathrm{E}$ de la version du 17 avril impose ainsi que le couteau ait des dimensions adaptées à la taille de l'animal prêt à être égorgé, que le fil, tranchant, ne soit pas endommagé ou encore que l'animal ne soit pas déplacé pendant un certain temps après la saignée. Si le contenu n'est pas strictement incompatible avec le rituel religieux (shekhita comme dhakât) tel qu'il est effectivement réalisé, le fait même pour un législateur de préciser son contenu est inédit. Ceci est aussitôt perçu comme une atteinte à la liberté religieuse.

L'annexe $\mathrm{E}$ soulève la réprobation de la Conférence des rabbins européens déjà navrée de ne pas avoir été consultée auparavant sur le contenu du texte ${ }^{25}$. Elle dépêche deux de ses membres dont un représentant permanent le grand rabbin de Bruxelles Albert Guigui, pour convaincre la Commission de faire prévaloir le principe de subsidiarité en la matière. Après plusieurs mois d'intense lobbying elle obtient la suspension de la proposition de la Commission ${ }^{26}$ par son président Jacques Delors,

\footnotetext{
${ }^{22}$ La Commission soumet une proposition législative dans le cadre des grandes orientations définies par le traité et le Conseil adopte la version finale de la législation après avis consultatif du PE et éventuellement du CES.

${ }^{23}$ Projet de proposition de règlement (CEE) COM (91)136 sur la protection des animaux au moment de leur abattage et de leur mise à mort, communication de M. Mac Sharry, DG Agriculture, VI/B.11.2, PVET/FR/0041.17 avril 1991.

${ }^{24}$ Cette étiquetage avait été réclamé dès 1985 par le Farm Animal Welfare Council (UK) dans son Report on the Welfare of Livestock when Slaughtered by Religious Methods.

${ }^{25}$ Lors d'un entretien réalisé à la synagogue de Bruxelles le 15 mai 2007, le grand rabbin de Bruxelles A. Guigui disait n'avoir été consulté qu'après la rédaction d'une première version du règlement initial. II raconte comment il a été alerté par un article du Soir (Belgique) qui rapportait les propos de Brigitte Bardot remerciant le gouvernement belge et la Commission européenne de leurs volontés respectives d'améliorer le sort des animaux au moment de l'abattage. II a contacté les services de la Commission laquelle a ensuite invité formellement la conférence des rabbins à donner son avis.

${ }^{26}$ A. Guigui: « Nous avons fait un travail extraordinaire, nous avons contacté tout le monde. [...] On a fait ce qu'on appelle une évidence scientifique, on a apporté des témoignages auprès du conseil vétérinaire supérieur
} 
"pour vérification des répercussions de la proposition sur les abattages rituels ${ }^{27}$. La version finale validée par l'ensemble des commissaires est publiée près de sept mois plus tard ${ }^{28}$. Elle ne contient plus qu'une seule référence à l'AR pour l'exclure totalement de son champ d'application ${ }^{29}$. Autrement dit, la Commission choisit de ne plus l'aborder et de laisser aux États membres la responsabilité de leur organisation.

\section{Le Parlement européen et le Conseil économique et social : traçabilité et harmonisation}

Le projet de règlement est ensuite examiné par le Parlement européen (PE) au titre de la procédure de consultation obligatoire, et par le Conseil économique et social (CES) selon la procédure de consultation optionnelle. Le PE demande l'étiquetage des produits issus de l'égorgement rituel, le CES réclame pour sa part l'harmonisation de ses pratiques, deux exigences que la Commission avait inclues dans la première version de sa proposition.

Le PE expose en préambule de ses amendements ce qu'il souhaite de ce "très attendu " règlement dont il salut l'engagement pour la recherche d'une meilleure protection des animaux d'abattage, qualifié d'objectif le plus important - sous-entendu plus important que les objectifs d'harmonisation sur le marché unique -. Le PE se place sur le terrain de la protection animale et de la défense du consommateur et insiste sur la nécessité d'informer afin « [...] que le consommateur qui achète de la viande le fasse en reconnaissance de la meilleure qualité de la viande et en sachant que les animaux ont été traités aussi bien que possible et étourdis avant d'être tués (?) ${ }^{30}$. II préconise l'étiquetage systématique de la viande issue d'un abattage selon un rite religieux ${ }^{31}$.

Le CES suggère de soumettre l'AR aux dispositions générales de protection des animaux, un encadrement plus étroit et souhaite que l'on envisage l'harmonisation de ses méthodes au niveau de l'Union. "S'agissant de la dérogation proposée dans le cas de méthodes spéciales d'abattage requises par certaines communautés religieuses, il conviendrait de préciser que les règles en matière d'acheminement et d'hébergement ne doivent pas s'écarter des dispositions générales de protection des animaux et que

qui conseille la Commission, il y avait Temple Grandin, le professeur Dantzer, le professeur Delhi. [...]. On a fait barrage [...] On a également été voir tous les commissaires de l'époque un par un. D'abord pour les sensibiliser car ils ne connaissaient pas le problème. [...] Et finalement le vote qui a eu lieu a été favorable, le vote des commissaires nous a donné raison à 11 sur 12 , une abstention, le seul qui a voté pour c'était lui. On a été au Parlement, on a été au Conseil, partout... c'est une histoire merveilleuse à raconter! Ça a créé un climat de paix depuis $1993 »$.

${ }_{27}$ Procédure écrite E/471/91 du 26 avril 1991, Source: SANCO (Direction générale "Santé et protection des consommateurs "). Le président de la Commission est soutenu par le commissaire chargé de la compétition, le britannique sir Leon Brittan (conservateur), l'allemand Peter Schmidhuber (CSU) commissaire au Budget, et le belge Karel van Miert (SP), commissaire en charge du transport et de la protection du consommateur.

${ }^{28}$ La proposition finale de règlement de la commission (COM(91)136 final), datée du 8 novembre 1991.

${ }^{29}$ Article $1^{\text {er }}$, alinéa 2 précise que le règlement «... n'affecte pas les dispositions nationales relatives aux méthodes spéciales d'abattage requises par certains rites religieux particuliers ».

${ }^{30}$ Report on the Commission proposal for a Council regulation on the protection of animals at the time of slaughter or killing (COM(91) 0136 final - C3-0449/91) rapporteur: David Morris. 26 June 1992. Source: Eurogroup for Animals, May 2007.

${ }^{31}$ Amendement au paragraphe 2 de l'article 1 : "In the latter case the meat on sale to the consumer should be clearly labelled". 
seules l'immobilisation et la mise à mort peuvent s'effectuer conformément aux principes religieux de ces communautés. Cela étant, il y a lieu d'examiner dans quelle mesure il serait possible, après avoir consulté les communautés religieuses concernées, de convenir au niveau communautaire de méthodes uniformes d'AR pour chacune d'entre elles ".

\section{Le COREPER ${ }^{32}$ et le Conseil : liberté religieuse et économique}

II faut attendre près de deux ans pour que le Groupe des experts agricoles vétérinaires se réunisse ${ }^{33}$ et propose au COREPER trois amendements d'importance : un changement de statut du « règlement " qui devient « directive "34, que l'AR soit couvert par la directive, et enfin que ni l'étourdissement, ni les obligations concernant l'immobilisation (annexe B), ni les techniques de saignée (annexe D) ${ }^{35}$ ne soient obligatoires. Toutefois des pays très impliqués sur ce sujet et qui souhaitent minimiser une concurrence jugée déloyale comme l'Irlande - qui procédait à cette époque à une mise à mort selon le rite religieux avec étourdissement préalable -, et la France qui s'impose des mesures strictes et coûteuses de contention de ces animaux se montrent hostiles à cette version de la directive ${ }^{36}$. Le Conseil réuni quelques jours plus tard rejette les amendements proposés par le Groupe. La majorité des délégations exprimées ainsi que la Commission plaident pour la reprise de la proposition initiale de la Commission ${ }^{37}$.

Le texte est renvoyé à nouveau au Groupe des experts agricoles et vétérinaires pour le COREPER ${ }^{38}$ lequel réitère sa proposition ${ }^{39}$. Les directeurs généraux et chefs des services vétérinaires appuient cette version, ajoutent une obligation de contention comme souhaité par la délégation française, ainsi qu'un article attribuant aux autorités religieuses la responsabilité de l'application du rite, et aux vétérinaires le contrôle des opérations non religieuses ${ }^{40}$. Le COREPER demande l'adoption de cette version à l'unanimité des États

\footnotetext{
${ }^{32}$ Comité des représentants permanents.

${ }^{33}$ Réunion des 29 et 30 avril puis 3 et 4 juin 1993

${ }^{34}$ Le règlement est directement et obligatoirement applicable en l'état par les États membres alors que la directive, plus flexible, laisse le choix aux États membres de la formulation adéquate dans leur législation nationale.

${ }^{35}$ Amendement à l'article 5 : «Les autorités compétentes des États membres peuvent déroger aux dispositions prévues au paragraphe 1 sous b), c) et d) en ce qui concerne les méthodes particulières d'abattage requises par certaines rites religieux. "

${ }^{36}$ État des travaux du Groupe des experts agricoles et vétérinaires, 7116/93, 7 Juin 1993 et Rapport de ce même Groupe daté du 8 juin 1993 (ref. 7152/93).

${ }^{37}$ Extrait du Procès Verbal de la $1672^{\circ}$ session du Conseil du 14 juin 1993 (sans.ref)

${ }^{38}$ État des travaux du Groupe des experts agricoles et vétérinaires, 19 novembre 1993 Doc n $10197 / 93$

${ }^{39}$ Ajout à l'article 5 d'un second paragraphe: «Pour les animaux faisant l'objet de méthodes particulières d'abattage requises par certains rites religieux. Exigences prévues au paragraphe $1^{\text {er }}$ sous c) ne sont pas d'application ». Les directeurs généraux/chefs des services vétérinaires (dans la demande française d'harmonisation des (coûteux) moyens de contentions est également incorporée en annexe B pour constituer la version définitive de la directive 93/119/CE adoptée par le Conseil.

${ }^{40}$ Annexe du Rapport des 2 et 3 et 7 décembre 1993 au COREPER n 10 759/93: «Toutefois, l'autorité religieuse de l'État membre pour le compte de laquelle les dits abattages sont effectués est compétente pour l'application et le contrôle des dispositions particulières applicables à l'abattage selon certains rites religieux. Cette autorité opère pour les autres dispositions sous la responsabilité du vétérinaire officiel, tel que défini à l'article 2 de la directive 64/433/CEE ».
} 
membres (sauf la France qui s'abstient) ${ }^{41}$. Le dénouement est rendu possible grâce à un article, qui prévoit que les États peuvent appliquer des dispositions " plus strictes » que celles prévues par la directive (art. 18), minimisant du même coup la portée de celle-ci. De règlement, la proposition est devenue une directive qui admet que les États peuvent éventuellement procéder comme ils le souhaitent. La présidence belge du Conseil de l'UE adopte cette directive extraordinairement consensuelle.

C'est donc sans y être formellement contraints - notamment grâce à cet article 18 -, que les États membres ont majoritairement adopté le principe dérogatoire et que l'AR sans étiquetage s'est généralisé privilégiant la domination des acteurs économiques sur le marché halal en particulier, lesquels ont toute latitude pour définir les conditions d'application du rite.

\section{Les raisons du compromis dans la directive de 1993, et celles de son obsolescence}

Au moins cinq raisons expliquent l'aboutissement à un tel compromis : le soutien des États aux petits producteurs sur un marché rituel artisanal localement organisé, la relative impuissance des groupes de protection animale à rendre leur discours audible par le grand public, le désintérêt des associations de consommateurs vis-à-vis de la question rituelle, l'assimilation des méthodes d'AR shekhita et dhakât, la volonté de contrôle des États sur le culte. Près de quinze années plus tard, la situation a évolué sur tous ces points et comme nous allons le voir, plusieurs arguments semblent militer pour une intervention plus aboutie de la puissance publique dans la régulation des produits rituels même si la façon d'intervenir, le périmètre ainsi que le niveau d'intervention national ou communautaire, restent encore largement à préciser.

\section{De la liberté à la stabilité sur le marché halal}

Le compromis de 1993 a bénéficié d'abord aux acteurs économiques engagés dans le marché rituel, et surtout aux petits producteurs. Une interdiction de l'AR, ou à l'inverse une autorisation de dérogation assortie d'une obligation d'étiquetage, auraient empêché le développement d'un précieux marché de dégagement et mis en danger de petites entreprises locales pour qui ce marché pouvait représenter un débouché important (Brisebarre, 1993 ; MAD, 1995 ; Bergeaud-Blackler, 2001). Pourquoi dans ces conditions brider ce marché discret auquel se raccrochaient quelques-uns des plus vulnérables producteurs déjà malmenés par certains volets de la PAC ${ }^{42}$ ? On n'anticipait pas, à la fin des années quatre-vingt, que l'offre et la demande de produits rituels croîtraient et que des poids lourds du négoce et de la distribution se substitueraient peu à peu aux petits fermiers ${ }^{43}$. La concentration de la production et le changement des structures productives dans le secteur de l'industrie carnée, la compétition internationale et les opportunités d'exportation, ont entraîné une augmentation nette de l'AR et un nombre croissant de carcasses rituelles dans les circuits de distribution conventionnels.

\footnotetext{
${ }^{41}$ Note 11 442/93 Note point du COREPER au Conseil, 17 décembre 1993.

${ }^{42}$ Politique agricole commune.

${ }^{43}$ Agriculture and Agri-Food Canada, Government of Canada (2007).
} 
Aujourd'hui la méfiance des consommateurs et le mécontentement des organisations de protection animale sont tels que les distributeurs et les industries de transformation réalisent que la stabilisation d'une norme religieuse sera tôt ou tard un passage obligé pour le développement du marché halal ${ }^{44}$. Les derniers obstacles proviennent surtout des acteurs de l'industrie de la viande, notamment en France où le marché intérieur halal est le plus important d'Europe. Adoptant une stratégie à courte vue, ils souhaitent garder l'avantage dont ils ont bénéficié jusqu'ici, conservant une attitude de repli sur soi qui contraste nettement avec le dynamisme de ce marché sur d'autres continents.

\section{Changement de stratégie des organisations de protection animale}

Dans les années quatre-vingt-dix en Europe, le fait est relativement bien admis que l'alimentation est affaire culturelle et que des exceptions à certaines réglementations sont acceptables tant qu'elles n'affectent que les consommateurs concernés. Les organisations de protection animale n'ont pas le soutien populaire pour imposer une interdiction sauf en Suisse et en Suède où ces abattages sont interdits depuis plusieurs décennies (Gurtman, 2005 ; Bergeaud-Blackler, 2005). Au début des années quatrevingt-dix, on observe une politisation de ce thème (Brisebarre, 1998) qui répond surtout aux politiques sécuritaires dans le traitement de l'immigration, et qui finalement conforte plutôt les positions antiracistes et antixénophobes des partis de gauche et environnementaux. À l'exception d'événements particuliers ${ }^{45}$, les associations de protection animale ne communiquent guère publiquement sur l'AR de crainte d'être taxées d'antisémitisme ${ }^{46}$. II est plus politiquement correct d'opposer aux sollicitations des organisations de protection animale un attachement aux valeurs de multiculturalisme, de citoyenneté et d'intégration comme cela a été très bien illustré chez les Verts en Allemagne (Smith, 2007).

Mais un changement est perceptible. Au Royaume-Uni où le lobby animal welfare est puissant, la mobilisation des associations de protection animale "au nom du consommateur " a commencé à devenir audible auprès du grand public notamment après les crises sanitaires et épizooties (ESB, foot and mouth). En plaçant le consommateur au centre des enjeux, les organisations britanniques ont sensiblement modifié les termes du débat. Elles ont pu en outre s'appuyer sur un rapport du très crédible $\mathrm{FAWC}^{47}$ qui demandait sans ambiguïté la suppression pure et simple de toute dérogation à l'étourdissement, libérant la parole des associations welfaristes qui ont pu mener un combat pour l'étiquetage systématique des produits issus de ces pratiques (Bergeaud-Blackler, 2004). Le Royaume-Uni est certainement le pays d'Europe où les débats publics sur ce sujet sont à la fois les plus vigoureux et les plus consensuels, ce qui s'explique par le dynamisme du commerce halal ${ }^{48}$, une grande tolérance vis-à-vis

\footnotetext{
${ }^{44}$ Lire notamment "Business is urged to see opportunity in Muslim community", The Times, May $21^{\text {st }}, 2007$.

${ }^{45}$ Abattages massifs lors de la fête musulmane du Sacrifice (quasi annuelle).

${ }^{46}$ Les liens de la présidente de la Fondation Bardot avec le FN, fortement médiatisés, ont pu renforcer cette forme d'autocensure chez les défenseurs des animaux.

${ }^{47}$ Farm Animal Welfare Council : organe indépendant établi par le gouvernement britannique en 1979.

${ }^{48}$ Halal Foods - UK (2002)
} 
de ces produits dans les lieux publics et notamment à l'école (parfois jusqu'à l'excès ${ }^{49}$ ), et par l'attention prêtée par les Britanniques aux questions de bien-être animal. Cela peut expliquer en partie pourquoi les initiatives pour tenter de faire du secteur halal, à la fois un commerce rentable, religieusement et éthiquement acceptables pour tous sont plus nombreuses dans ce pays qu'ailleurs en Europe. Le temps où il était fait appel au vétérinaire issu d'une minorité musulmane pour convaincre les fidèles de renoncer à l'AR y semble révolu, remplacé par celui de l'engagement de débats à des niveaux institutionnels avec les acteurs religieux, ceux de la production et de la régulation. L'adhésion, encore timide, des musulmans à ce dialogue reste toutefois subordonnée au renoncement par les organisations de protection animale à ces campagnes émotionnelles orchestrées chaque année lors de la fête musulmane du sacrifice par le biais de vidéo sanglantes diffusées sur Internet ou de récits repris par certains médias. II dépend également du traitement politique, social et médiatique de l'Islam, ressenti comme partial par les intéressés en particulier depuis les attentats du 11 septembre 2001 à New York et Washington et ceux du 7 Juillet 2005 à Londres.

\section{L'objectif de protection du consommateur}

Placer le consommateur au centre des discussions a constitué un tournant dans la stratégie britannique visant à rendre public le débat sur l'AR. Pourtant, à quelques exceptions près, les associations de consommateurs restent muettes sur ce sujet. II faut dire qu'à l'échelon européen, la capacité de mobilisation de ces associations est relativement faible notamment en raison de la disparité des situations entre Nord et Sud de l'Europe. Au Sud, les marges d'action sont limitées en raison du faible nombre, du manque de moyen et de légitimité des organisations représentatives de consommateurs. La situation est plus favorable en Europe du Nord où les associations de consommateurs sont plus structurées, soutenues et consultées par les États, et où elles bénéficient d'une plus grande attention des opinions publiques (Lamarthe, 2001). Cette hétérogénéité entre Nord et Sud est problématique pour les fédérations de consommateurs ${ }^{50}$ car l'échelle de mobilisation la plus efficace pour la protection du consommateur est celle des échanges commerciaux, c'est-à-dire I'UE et plus largement l'OMC ${ }^{51}$. En plus de cette faiblesse structurelle, le problème du droit des minorités religieuses n'est pas en bonne place sur leur agenda, si ce droit y a une place. Pour être efficace, il faut que ces associations trouvent en face d'elles des institutions attentives à leurs thèmes de mobilisation ce qui ne s'est vraiment réalisé au niveau des institutions communautaires que très récemment. À l'époque de la fabrication de la directive de 1993, la Commission Delors comportait une curieuse direction générale " du transport et de la protection du consommateur ». Le consensus qui a constitué en une rupture dans l'orientation de la stratégie communautaire de protection du consommateur n'a été réalisé qu'après la période 1996-1999, marquée

\footnotetext{
${ }^{49}$ Une école britannique avait remplacé la traditionnelle dinde de Noël par du poulet halal, pour que les musulmans (un cinquième de la population de l'école) puisse partager le repas. Cette décision avait provoqué un tollé chez les parents qui ont finalement obtenu le retour de la dinde au menu. Paul Stokes (2006), "School U-Turns on Halal Christmas Menu", Telegraph, 18 November.

${ }^{50}$ Comme le BEUC à Bruxelles par exemple.

${ }^{51}$ Organisation mondiale du commerce.
} 
par l'affaire de l'ESB ${ }^{52}$ et les premières mobilisations contre les OGM ${ }^{53}$ (Bergeaud \& Feretti, 2006). La "brutale multiplication des mobilisations" autour des novel foods a permis une unité de vue sur la nécessité d'un étiquetage alimentaire de la part de protagonistes aux intérêts divergents (Granjou, 2004).

Aujourd'hui le regroupement sous la houlette d'une direction générale de la Santé et de la Protection du consommateur, de la Sécurité alimentaire, de la Santé publique, de la Santé et de la Protection animale, offrant une plus grande résistance aux pressions des puissantes DG, comme la DG Agriculture, présente une configuration un peu plus favorable aux consommateurs. Mais surtout, la réflexion menée depuis une dizaine d'années sur l'étiquetage des aliments posant des problèmes d'ordre éthique peut aujourd'hui permettre d'aborder avec de nouveaux outils intellectuels celle sur le statut, l'identification et l'accessibilité des produits rituels à l'échelle de l'UE.

\section{Peut-on traiter de façon identique dhakât et shekhita?}

L'absence de comité de consultation des organisations religieuses auprès de la Commission, le positionnement intransigeant de certains lobbies religieux et la faiblesse d'autres ont influé sur les choix opérés dans la directive de 1993. Les communautés juives d'Europe, représentées par une seule organisation (la Conférence des rabbins européens), ont défendu le droit à la Shekhita, pour des raisons matérielles, organisationnelles autant que de principe. Dans le discours et la pratique politique antisémites européens, la Shekhita occupe une place de choix (Erlanger, 2006; Krauthammer, 2000) ce qui explique que toute remise en question de l'AR leur soit vécue comme douloureuse. L'autre raison tient dans le rôle majeur du système de la cacheroute dans l'autofinancement de la communauté religieuse. En l'état actuel des relations entre États et cultes, les institutions juives ont le sentiment que remettre en cause ou limiter la Shekhita réduirait leur autonomie, affaiblirait leur capacité à se structurer, l'organisation de la cacheroute étant presque toujours liée à celle de l'autorité et du pouvoir (Bauer, 1996). C'est pourquoi lors de la rédaction de la directive de 1993, la Conférence des rabbins européens n'a pas ménagé ses efforts pour obtenir la reconnaissance de l'AR dans le $6^{e}$ considérant de la directive stipulant qu'il est "nécessaire » de "prendre en compte les exigences particulières des rites religieux ».

Aujourd'hui sa détermination et sa capacité à se faire entendre se retournent un peu contre elle, car les acteurs musulmans du marché halal s'appuient sur cette dérogation accordée à la communauté juive pour la revendiquer à leur tour, alors même que la question n'est pas tranchée au sein des autorités religieuses islamiques. Les rabbins craignent ainsi que le débat qui agite les communautés musulmanes atteigne à leur tour les communautés juives d'Europe, qu'il mette en péril le fragile consensus trouvé avec les États et l'UE. Les rabbins ont toujours été et restent favorables à une intervention minimale de l'État. Les responsables musulmans ont besoin de la puissance publique pour limiter l'emprise des stratégies de marketing sur les signes et symboles religieux, emprise qui épuise leur capacité à se fédérer et s'institutionnaliser. Ne faudrait-il pas traiter de manière différenciée le problème de la shekhita et du dhakât? La question

\footnotetext{
${ }^{52}$ Encéphalopathie spongiforme bovine.

${ }^{53}$ Organismes génétiquement modifiés.
} 
mérite d'être posée aujourd'hui. Enfin, la volonté des institutions européennes d'encourager le dialogue avec les religions (Massignon 2002, 23) est également un élément de changement important depuis 1993, propre à faciliter un réexamen des conditions industrielles du dhakât. Reste ensuite à savoir si un consensus européen serait suffisant alors que les courants d'influence islamique proviennent du MoyenOrient et les normes de fabrication halal d'Asie du Sud-Est (Malaisie, Indonésie, etc.).

\section{De l'ordre public à la lutte contre la discrimination}

Comme le résume Bérengère Massignon « l'attachement à un modèle spécifique de relations Églises/État est un des éléments de l'identité et de la culture politique nationales ${ }^{54}$. Dans certains États cette relation est aussi une relation de défiance mutuelle notamment en raison du caractère international des cultes qu'une nation peut percevoir dans certaines circonstances comme menaçant. En France, contrôler l'AR est un moyen de surveiller les finances du culte, éventuellement se choisir un interlocuteur. Dans ce pays où la centralisation napoléonienne du consistoire a été rendue possible par celle de la cacheroute, permettant à l'État de n'avoir qu'un interlocuteur représentatif de la communauté juive de France (Nizar-Benchimol, 1997, 222), il est admis que la gestion administrative et politique de l'AR constitue une ingérence de l'État dans les affaires religieuses et qu'elle se justifie pour des motifs d'ordre public ${ }^{55}$. C'est également pour ces motifs qu'au moment de la montée du FIS ${ }^{56}$ en Algérie, d'attaques terroristes islamiques dans l'Hexagone que le ministre de l'Intérieur tentait, sans finalement y parvenir, d'accorder à la Grande mosquée de Paris le monopole du contrôle de la production et de la commercialisation de la viande halal (BergeaudBlackler, 2001). Au moment de la rédaction de la directive, il était possible d'imaginer contrôler les ressources d'une " cacheroute islamique française ». Mais le marché de la viande rituelle n'a pas cessé d'évoluer depuis sa mutation, il s'est mondialisé, diversifié, ses structures de production et de distribution se sont transformées, et l'idée d'obscurs liens entre marché de la viande halal et financement d'organisations terroristes aux ramifications internationales s'est peu à peu dissipée.

Désormais les États sont interpellés afin de garantir la liberté du culte et notamment l'accès aux aliments halal et casher dans les lieux publics ${ }^{57}$. Même si les situations sont assez inégales dans les pays de l'Union, les réponses des États ont été plutôt non interventionnistes évitant ainsi les critiques d'ingérence dans les affaires religieuses. Pourtant l'exemple des États-Unis montre que réguler ne signifie pas encourager ou

\footnotetext{
${ }^{54}$ Cha'are Shalom ve Tsedek c. France, Arrêt de la Cour européenne des Droits de l'Homme du 27 juin 2000, Strasbourg après Requête $n^{\circ} 27417 / 95$, Conseil de l'Europe.

${ }^{55}$ Front islamique du salut.

${ }^{56}$ Dassetto Felice, Ferrari Silvio et Maréchal Brigitte (2007), Islam in the European Union: What's at Stake in the Future?, Bruxelles, European Parliament, Policy Department Structural and Cohesion Policies, Culture and Education, May, [http://www.libertysecurity.org/IMG/pdf_Islam_in_Europe_EN.pdf]. Également, Les musulmans au sein de I'UE : discrimination et islamophobie (2006), EUMC-Observatoire européen des phénomènes racistes et xénophobes, [http://1001nights.free.fr/textes/Manifestations_FR.pdf].

57 "The observance of the rules on religious freedom requires that Muslims (and the members of other religions) may be able to obtain, in the canteens of public institutions, food not forbidden by their religion. This is the minimum level below which it is not possible to go without violating Art. 9 of the European Convention", (Dassetto, Ferrari \& Maréchal, 2007).
} 
dissuader mais plutôt établir des limites pour parvenir à une division claire des responsabilités. L'absence de transparence sur les marchés d'aliments rituels est très certainement responsable de la faible ou nulle accessibilité des aliments halal ou casher dans les lieux publics en Europe. Des juristes en déduisent aujourd'hui que cette situation constitue une violation de l'article 9 de la Convention, car la liberté religieuse n'est pas assuré $^{58}$. Cette conclusion peut se discuter mais au moins la question est posée. Le plus préoccupant selon nous est que la situation présente conduit, dans le cas du halal en particulier, à une radicalisation des signes et symboles religieux, à une surenchère du rituel, à une instrumentalisation raciste, antisémite et islamophobique des préoccupations légitimes des associations de protection animale. Dans une Union européenne politique inachevée, les droits des consommateurs à choisir et à être informés ne sont pas uniquement des droits individuels, ils contribuent bon gré mal gré à forger une identité citoyenne européenne. Fermer les yeux sur les droits de certains d'entre eux en invoquant le principe de non-intervention des États et la liberté religieuse est un contresens. La révision de la directive de 1993 est l'occasion d'engager un nouveau débat sur le statut légal de ces aliments rituels, au cœur des relations complexes entre État, religion, immigration et mondialisation de l'économie.

A qui est destiné l'abattage rituel et qui doit dire en quoi il consiste ? Étonnamment, ces deux questions restent encore en suspens. Au cours des trois dernières décennies l'abattage rituel industriel en Europe a accompagné en douceur la concentration de la production et le changement des structures productives dans le secteur de l'industrie carnée, la compétition internationale et les opportunités d'exportation. Par sa visibilité notamment au moment de l'Aid el Kebir, il a permis une mise en scène de la cruauté bien utile à la mobilisation des partisans de la cause animale. Le traitement légal indifférencié des abattages musulmans et juifs a permis une meilleure rentabilité de l'abattage industriel. Certains États ont utilisé la gestion de l'autorisation de procéder au sacrifice comme un instrument de contrôle du champ religieux. Comme nous avons tenté de le montrer à l'aide de nombreux exemples, cette gestion de l'abattage rituel est peu viable dans un contexte de globalisation des échanges alimentaires où le consommateur et ses choix individuels éthiques ont une influence croissante sur le marché et les instruments qui régulent celui-ci. Si la sécularisation constitue bien ce " processus par lequel une société cesse progressivement de faire appel à la notion de sacré et à la volonté ou à la révélation divines dans la gestion des questions temporelles et notamment politiques " (Hermetand alii, 2001), alors elle semble bien être entrain de faire tomber les grand murs des abattoirs européens, ces derniers territoires du sacré dans lesquels des acteurs aux objectifs divergents ont pu construire autour de leurs intérêts particuliers un système opaque dans un espace gris entre religieux et politique que plus personne ne pensait avoir la compétence légitime de contrôler. La recherche de l'efficacité économique conduira-t-elle l'Union Européenne à prendre acte de cette transformation, à clore le débat qu'elle avait amorcé dès 1990 et participer à la sécularisation encore inachevée des abattoirs? 


\section{BIBLIOGRAPHIE}

Bauer Julien (1996), La nourriture cacher, $1^{\text {re }}$ éd., Paris, PUF, coll. «Que sais-je, 3098 ».

Bergeaud-Blackler Florence (2001), « La viande halal peut-elle financer le culte musulman ? », Le journal des anthropologues : anthropologie et économie, 84, p. 145-171.

Bergeaud-Blackler Florence (2004), « Nouveaux enjeux autour de l'abattage rituel: une perspective européenne », Cahiers d'économie et de sociologie rurales, 73, p. 5-33.

Bergeaud-Blackler Florence et Ferretti Maria-Paola (2006), "More Politics, Stronger Consumers? A New Division of Responsibility for Food in the European Union", Appetite, 47 (2), p. 134142.

Brisebarre Anne-Marie (1993), "The Sacrifice of 'Id al-kabir. Islam in the French Suburbs", Anthropology Today, 9(1), February, p. 9-12.

Brisebarre Anne-Marie (1998), La fête du mouton : un sacrifice musulman dans l'espace urbain, Paris, CNRS Éditions, coll. "Méditerranée ».

Erlanger Simon (2006), Jewish Political Studies Review, 18 (1-2, The Politics of 'Transmigration': Why Jewish Refugees Had to Leave Switzerland from 1944 to 1954), Spring.

Hermet Guy, Badie Bertrand, Birnbaum Pierre, Braud Philippe (2005), Dictionnaire de la science politique et des institutions politiques $\left(6^{\circ} \mathrm{Ed}\right.$.)

Granjou Céline (2004), “Traçabilité, étiquetage et émergence du "citoyen-consommateur" : l'exemple des OGM », Chatriot Alain, Chessel Marie-Emmanuelle et Hilton Matthew, $A u$ nom du consommateur : consommation et politique en Europe et aux États-Unis au $X X^{e}$ siècle, Paris, la Découverte, coll. «L'Espace de l'histoire », p. 199-211.

Gutman Benjamin N. (1999), "Ethical Eating: Applying the Kosher Food Regulatory Regime to Organic Food", The Yale Law Journal, 108 (8), June, p. 2351-2384.

Krauthammer Pascal (2000), Das Schächtverbot in der Schweiz 1854-2000, Zurich, Schulthess.

Lamarthe Béatrice (2001), La défense du consommateur dans l'Union européenne, Paris, la Documentation française, coll. «Réflexe Europe ».

Marei Mohamed H. (2001), "A Rising Star?: Halal Consumer Protection Laws", Working Paper, Harvard Law School.

Massignon Bérengère (2002), “ Les relations des organismes européens religieux et humanistes avec les institutions européennes: logiques nationales et confessionnelles », Commissariat général du Plan-Institut universitaire de Florence, Croyances religieuses, morales et éthiques dans le processus de construction européenne, Paris, la Documentation française, p. 23-40.

Masoudi Gerald (1993), "Kosher Food Regulation and the Religion Clauses of the First Amendment", The University of Chicago Law Review, 60 (2) Spring, p. 667-696.

Nizar-Benchimol Sophie (1997), L'Économie du Croire: une anthropologie des pratiques alimentaires juives en modernité, Thèse de doctorat en Sociologie, Paris, EHESS.

Smith David (2007), "'Cruelty of the Worst Kind': Religious Slaughter, Xenophobia, and the German Greens", Central European History, 40(1), p. 89-115.

Wigoder Geoffrey et Goldberg Sylvie Anne, Gillet Véronique, Serandour Arnaud et Veyret Gabriel R. (1996), Dictionnaire encyclopédique du judaïsme, Paris, Éditions du Cerf/R. Laffont, coll. « Bouquins».

Willaime Jean-Paul (2004), Europe et religions : les enjeux du xxi siècle, Paris, Fayard, coll. «Les Dieux dans la cité : religions et sociétés ». 


\section{Rapports}

Bergeaud-Blackler Florence (2001), Enquête sur la consommation maghrébine de viande halal et sur les marchés de viande de boucherie halal en Aquitaine, rapport pour Aquibev, DRAF Aquitaine, ministère de l'Agriculture/DGAL, octobre.

Harrison Richard (ed.) (1985), Report on the Welfare of Livestock when Slaughtered by Religious Methods, Ministry of Agriculture, Fisheries and Food/Farm Animal Welfare Council.

Nefussi Jacques (dir.) (1996), Le marché de la viande halal en France, Mission Agro Développement rapport pour la DGAL ministère de l'Agriculture, Interbev.

2006 Report on International Religious Freedom (2006), Rapport annuel publié par le Bureau of Democracy, Human Rights and Labor, du département d'État (US). [http://www.state.gov/g/drl/rls/irf/2006/]

CSPI-Center for Science in the Public Interest, Silverglade Bruce, Farzan Leila, Ringer Heller llene, Soltis Cassandra et Kemeya Misa (1998), Food Labelling for the $21^{\text {st }}$ Century: $A$ Global Agenda for Action, May, [http://www.cspinet.org/reports/coindex.htm].

Agriculture and Agri-Food Canada, Government of Canada (2007), Global Halal Food Market, July, [http://www.ats.agr.gc.ca/africa/4352_e.pdf].

Halal Foods - UK - January 2002- (c) Mintel International Group Limited.

\section{Autres publications}

Buying Halal Food, brochure publiée par le New Jersey Office of the Attorney General, Division of Consumer Affairs, Office of Consumer Protection, Kosher Food Enforcement Unit [www.NJConsumerAffairs.gov]. [http://www.state.nj.us/lps/ca/brief/halal.pdf].

Buying Kosher Food, "Regulations Governing the Sale of Food Represented as Kosher", brochure publiée par le New Jersey Division of Consumer Affair. (NJAC 13:45A-21.1 et seq.)

[http://www.njconsumeraffairs.gov/brief/kosher.pdf]. 